\title{
MICROWAVE TISSUE PROCESING IN A TEACHING LABORATORY
}

R. S. Demaree, Jr.

Department of Biological Sciences, California State University, Chico, CA 95929

Many investigators have used microwave ovens for various aspects of biological sample processing for light microscopy (LM), transmission electron microscopy (TEM.) and scanning electron microscopy (SEM.) ${ }^{1}$

Microwave-assisted processing also can play a significant role in the teaching laboratory. Students routinely prepare samples for SEM in a single 3 hour laboratory period. They fix, dehydrate and dry with hexamethyldisilazane in less than $1 \frac{1}{2} 2$ hours. The only part of the process not utilizing microwave assist is the final 15 minute drying step in a conventional oven (Fig. 1.).

TEM samples may now be processed in a 3 hour laboratory period (Fig. 2.) We routinely fix, dehydrate and infiltrate samples during one laboratory period. Embedding molds are placed in a conventional drying oven until the next laboratory period. Blocks are trimmed, sectioned and viewed during the next laboratory session.

Lengthy immunological staining protocols requiring one or more days to perform may now be completed in a single laboratory session with microwave assist. A bench protocol for LM requiring more than 12 hours is now routinely completed in 2 hours (Fig. 3.) The final example is a TEM immunological label using microwave assist (Fig. 4.) The initial fixation through infiltration was done in a single lab period; blocks were then placed in a conventional oven overnight. The next day, ultrathin sections were immunolabelled in less than 2 hours.

In our hands, microwave-assisted biological sample processing is our primary method of sample preparation. All micrographs presented here are student projects. ${ }^{2}$

\section{References}

1. R. T. Giberson and R. S. Demaree, Jr. Microwave Techniques and Protocols, Totowa, NJ, Humana (2001).

2. Appreciation is expressed to Norm Fox, Charles Heise, Teresa Munoz and Julie Janes for use of their micrographs. 


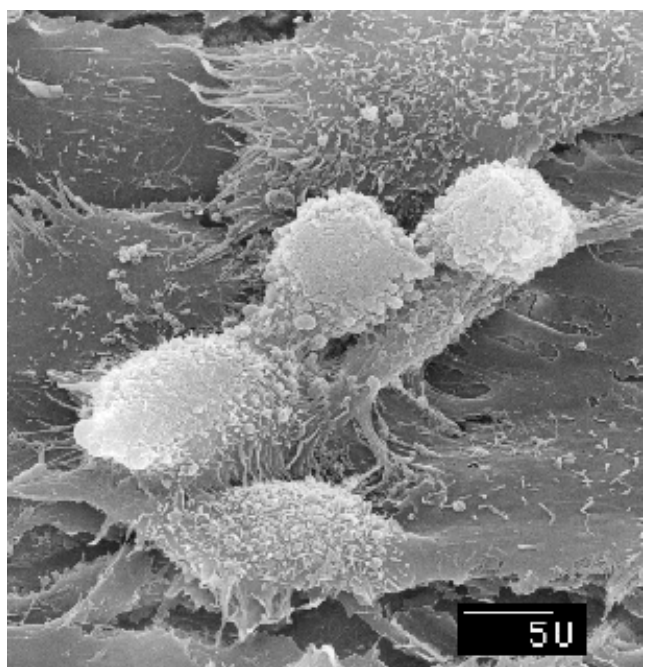

Microwave SEM prep of MODE-K cells prepared is less than 11/2 hours. Bar equals 5 micrometers.

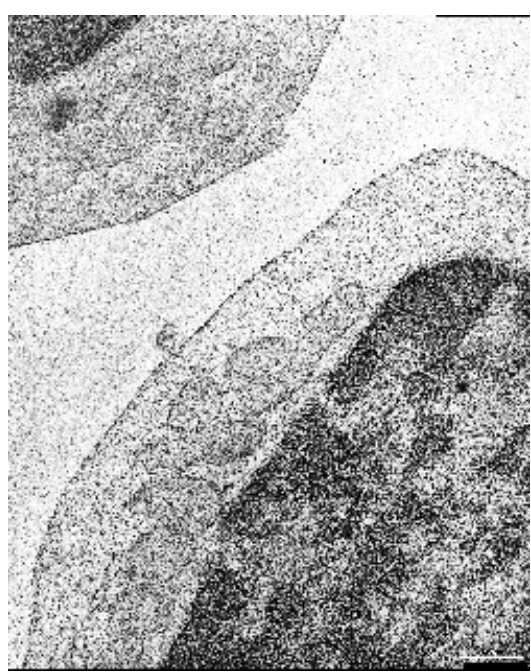

Microwave TEM prep from fix to plastic in a single 3 hour lab session. Bar equals 0.5 micrometers.

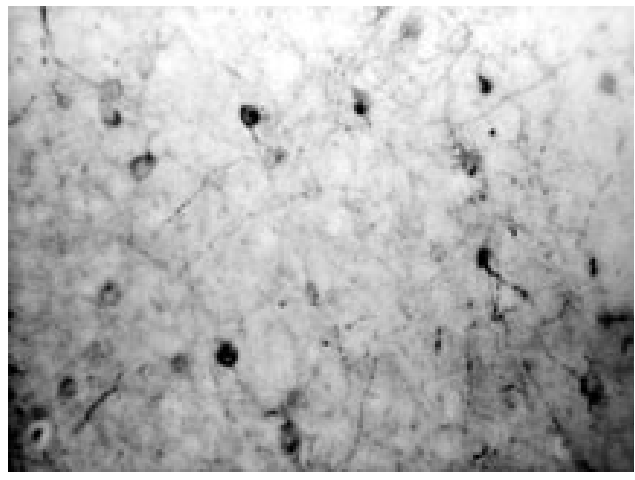

Microwave LM immuno label (GluR1) of frozen rat brain section in 2 hours. Normal staining time 12 hours. X 25 .

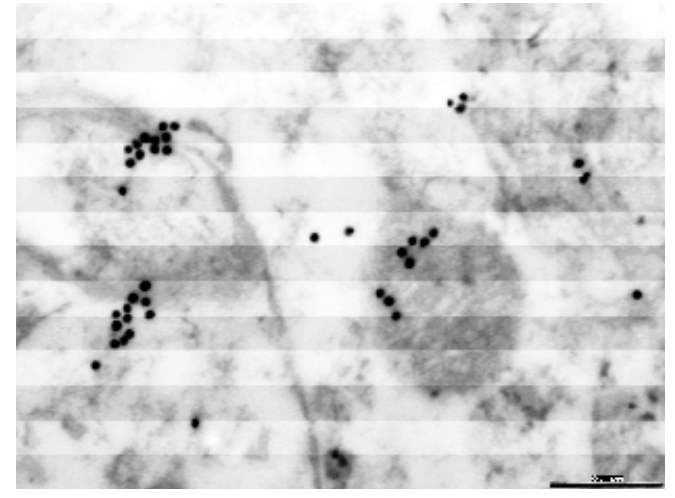

Microwave TEM immuno label (GluR1) completed in two 3 hour lab periods. Bar equals 0.4 micrometers. 